\title{
Administración de costos en las pymes de transformación en la región Laja Bajío de México.
}

[Artículos de investigación]

\author{
Christian Paulina Mendoza Torres ${ }^{* *}$ \\ María Campos Mendoza ${ }^{* * *}$
}

Recibido: 07 de enero de 2020

Revisado: 27 de junio de 2020

Aceptado: 10 de agosto de 2020

Cómo citar este artículo:

Mendoza, C. P. y Campos, M. (2020). Administración de costos en las pymes de transformación en la región Laja Bajío de México. Signos, Investigación en sistemas de gestión, 13(1). https://doi.org/10.15332/24631140.6340

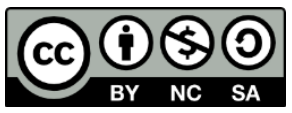

\section{Resumen}

Esta investigación se desarrolló con el objetivo de determinar si los empresarios de pequeñas y medianas empresas (pymes) de transformación en Celaya (Guanajuato, México) y la región Laja Bajío

\footnotetext{
* Artículo de resultado de investigación

** Universidad de Celaya. Doctorado en Administración, con especialidad en Desarrollo Organizacional. Magíster en Administración, con especialidad en Finanzas. Licenciada en Ciencias de la Comunicación, con especialidad en Investigación Social. Línea de investigación: Desarrollo Organizacional de las Pymes y Metodología de la Investigación. Universidad de Celaya, Celaya, México. Correo electrónico: pau_mendoza@hotmail.com, pmendoza@udec.edu.mx. ORCID: https://orcid.org/0000-0002-2998-714X

${ }^{* * *}$ Universidad de Celaya. Doctorado en Administración, con especialidad en Finanzas. Magíster en Fiscal. Licenciada en Contabilidad. Línea de investigación: Contabilidad de Pymes. Universidad de Celaya, Celaya, México. ORCID: https://orcid.org/0000-00030434-8402
} 
tenían establecidos controles para administrar adecuadamente sus costos de producción, analizar los elementos que los conforman y definir los controles que requieren para administrar eficientemente sus costos. El estudio inició con un enfoque exploratorio y terminó siendo explicativo bajo una metodología cuantitativa. Se elaboró y aplicó una escala de Likert a 42 empresas de transformación. Se determinó que los empresarios de estas pymes no cuentan con procedimientos administrativos necesarios para controlar sus costos de producción. Las pymes buscan pagar sus impuestos, pero no analizan los resultados obtenidos a la luz de las obligaciones que establecen las leyes fiscales de México. Este artículo expone la información necesaria para promover su reflexión y sobrevivencia.

Palabras clave: costos, costos de transformación, pymes, administración de pymes.

\section{Cost management in processing SMEs in the Laja Bajío region of Mexico}

\section{Abstract}

This research was conducted with the purpose of determining whether the entrepreneurs of small and medium processing companies (SMEs) in Celaya (Guanajuato, Mexico) and the Laja Bajio region had put in place controls to adequately manage their production costs, analyze the elements making up those costs and define controls required to efficiently manage their costs. The study was initially exploratory and ended up being explained under a quantitative methodology. A Likert scale was developed and applied to 42 processing companies. It was determined that those SMEs entrepreneurs have not implemented the necessary administrative procedures to control their production costs. SMEs seek to pay their taxes but fail to analyze the results obtained in light of the obligations established by Mexico's tax laws. This article presents the necessary information to promote their reflection and survival. 
Keywords: costs, processing costs, SMEs, SMEs management.

\section{Gestão de custos em PMEs de transformação na região de Laja Bajío, no México}

\section{Resumo}

Esta pesquisa foi realizada com o intuito de determinar se os empresários das pequenas e médias empresas de transformação (PMEs) de Celaya (Guanajuato, México) e da região de Laja Bajío tinham estabelecido controles para gerir adequadamente seus custos de produção, analisar os elementos que os compõem e definir os controles necessários para gerir eficientemente seus custos. O estudo teve um foco exploratório inicial e acabou sendo explicativo sob uma metodologia quantitativa. Foi desenvolvida uma escala Likert que foi aplicada a 42 empresas de transformação. Determinou-se que os empresários dessas PMEs não possuem os procedimentos administrativos necessários para controlar seus custos de produção. As PMEs procuram pagar seus impostos, mas não analisam os resultados obtidos em função das obrigações estabelecidas pelas leis fiscais do México. Este artigo apresenta as informações necessárias para fomentar sua reflexão e sobrevivência.

Palavras-chave: custos, custos de transformação, PMEs, gestão de PMEs.

\section{Introducción}

Las pymes son el eslabón fundamental para el crecimiento de México y la columna vertebral de la economía nacional por su alto impacto en la generación de empleos y en la producción nacional. De acuerdo con datos del Instituto Nacional de Estadística y Geografía (Inegi, 2019), las pymes representan el $99.8 \%$ de las unidades empresariales, generan el $52 \%$ del PIB y el 72 \% del empleo en el país. Sin embargo, muchas nacen sin un plan de negocios, basado principalmente en la administración de sus 
costos, que es esencial para que cualquier empresa sea eficiente, eficaz, productiva, innovadora y orientada hacia la calidad. Las organizaciones con una deficiente o nula planeación de costos difícilmente pueden aspirar a ser mejores, puesto que su preocupación principal consiste en mantenerse en el mercado y dejan de lado las posibilidades de crecimiento al no optimizar todos sus recursos. Esto se debe a que los costos requieren información; hay que obtener datos, registrarlos e informarlos. Pero todo ese esfuerzo resulta inútil si los datos no se analizan ni se utilizan realmente como una herramienta para alcanzar los objetivos fundamentales de la empresa. Como señala Calleja (2018), "muchos estudiosos de estos temas consideran que las empresas exitosas de ahora en adelante serán aquellas que sepan administrar con talento sus costos, o mejor aún, la información derivada de ellos” (p. 5).

Guizar (2007) afirma que hay seis obstáculos a los que se enfrentan los hombres de negocios en las pequeñas y medianas empresas, entre los que se destacan la escasez de capital de trabajo para la compra de maquinaria y equipo; la carencia de medios de producción e insumos indispensables para la continuidad de la producción; la falta de asesoría técnica que difunda los elementos y adelantos susceptibles de ser incorporados al proceso de producción, y la resistencia al cambio.

Podría pensarse que este último es el más importante para los empresarios cuando se trata de implementar mejoras o propuestas en la administración de los costos en las pymes, ya que la resistencia al cambio es muy fuerte, sobre todo en empresarios que piensan que si su forma de trabajar les ha funcionado bien, ¿para qué hacer cambios? Sin embargo, en estos obstáculos no se menciona la falta de control en sus operaciones productivas. La falta de control en sus sistemas de costos les impide conocerlos adecuadamente. 
A partir de 2014, la reforma fiscal obliga a todos los contribuyentes, incluyendo las micro, pequeñas y medianas empresas (mipymes), a llevar contabilidad electrónica. Sin embargo, muchos microempresarios ni siquiera saben utilizar los medios informáticos computarizados y se están orientando hacia la informalidad, sin considerar los controles administrativos necesarios para crecer o subsistir. Rodríguez (citado por Ojeda, 2013) indica que "la necesidad de conocer el funcionamiento de las organizaciones lleva implícita la descripción del ambiente donde se desarrolla la actividad de los individuos" (p. 24).

Las pymes constituyen una opción de desarrollo económico y social por su alta contribución a la generación de empleos, diversificación de la oferta de bienes y servicios, mejor equilibrio en la distribución del ingreso y logro de mayores índices de competitividad, al ser parte de las cadenas productivas y aportar el 50 \% de los empleos (Banco Mundial, 2016). Por esta razón, se debe reconocer la importancia de una adecuada administración de los costos.

Cuando se busca mejorar en las empresas, como señalan Hodgson y White (2002), "lo fundamental es adoptar la actitud apropiada para afrontar los asuntos y los problemas que encuentran como dirigentes y líderes de sus empresas" (p. 6). Para crear conciencia en los empresarios respecto a la importancia que tiene la administración de costos, esta investigación buscó determinar si los empresarios de pequeñas y medianas empresas (pymes) de transformación en Celaya (Guanajuato, México) y la región de Laja Bajío tienen establecidos controles que les permitan administrar adecuadamente sus costos de producción, con el fin de analizar los elementos que los conforman y definir los controles que deben establecer para administrar eficientemente sus costos.

Toda pequeña o mediana empresa debe contar con un sistema de costos que logre, en principio, determinar correctamente el costo de producción, 
con miras a establecer un sistema de información que propicie una cultura competitiva (Ramírez, 2018).

\section{Metodología}

La investigación tiene un alcance inicial exploratorio y posteriormente avanza hasta ser explicativa, pues buscó investigar si las pymes de transformación cuentan con procedimientos sistematizados de administración de costos e investigar cómo se conforma el costo de producción.

Se realizó un estudio cuantitativo orientado al análisis de los factores relacionados con la administración de los costos de las pymes en Celaya (Guanajuato, México) y su zona de influencia (Laja Bajío). El análisis se centró en examinar los elementos que inciden en el costo de producción de las pymes de transformación.

\section{Población y muestra}

El universo del estudio consideró la inclusión de empresas con las siguientes características:

- Empresas del sector industrial (sin considerar subsector para no limitar la muestra)

- $\quad$ Antigüedad mínima de 3 años

- Ventas en el ejercicio inmediato anterior superiores a 3 millones de pesos mexicanos

- Mínimo de 10 trabajadores y máximo de 250

La tabla 1 presenta la distribución de las pymes de Celaya y Laja Bajío que constituyeron la población total de esta investigación, con base en información proporcionada por el Inegi (2019). 
Tabla 1. Población de pymes analizadas en Celaya y Laja Bajío

\begin{tabular}{|l|l|l|l|l|l|l|}
\hline \multirow{2}{*}{ Cantidad de trabajadores } & \multicolumn{7}{c|}{ Municipios } & \multirow{2}{*}{ Total } \\
\cline { 1 - 7 } & $\begin{array}{c}\text { Apaseo El } \\
\text { Alto }\end{array}$ & $\begin{array}{c}\text { Apaseo El } \\
\text { Grande }\end{array}$ & Celaya & Cortázar & Villagrán & \\
\hline De 11 a 30 & 7 & 6 & 102 & 6 & 4 & 125 \\
\hline De 31 a 50 & 1 & 3 & 22 & 0 & 0 & 26 \\
\hline De 51 a 100 & 0 & 1 & 19 & 5 & 3 & $\mathbf{2 8}$ \\
\hline De 101 a 250 & 0 & 6 & 18 & 3 & 4 & $\mathbf{3 1}$ \\
\hline Total & $\mathbf{8}$ & $\mathbf{1 6}$ & $\mathbf{1 6 1}$ & $\mathbf{1 4}$ & $\mathbf{1 1}$ & $\mathbf{2 1 0}$ \\
\hline
\end{tabular}

Fuente: Inegi (2019).

Por circunstancias de acceso, confianza de los empresarios y conveniencia, la muestra fue aleatoria simple, de casos-tipo, y estuvo conformada por 42 pymes pertenecientes al sector manufacturero, consideradas sólidamente establecidas, debido a que la mayoría de ellas cuentan con 10 o más años de antigüedad. Son empresas cuyas ventas se ubican entre 1-6 millones de pesos mexicanos y cuyo número de empleados (en el 70 \% de los casos) es menor de 50. El perfil de entrevistados fue de nivel organizacional medio y alto, es decir, dueños, jefes de producción, contadores, gerentes y gerentes generales.

\section{Hipótesis y variables}

Hipótesis nula (Ho)

Las pymes que se ubican en la ciudad de Celaya y su zona de influencia no cuentan con un procedimiento sistemático de administración de costos.

\section{Hipótesis alternativa (Ha)}

Las pymes que se ubican en la ciudad de Celaya y su zona de influencia cuentan con un procedimiento sistemático de administración de costos.

Si la empresa está más profesionalizada y cuenta con alguna ayuda de un especialista en contabilidad, el procedimiento rústico, empírico y de acuerdo con las circunstancias se puede ir haciendo más metódico, 
ordenado, preciso con una secuencia lógica que permita obtener resultados más confiables y libres de errores.

La pregunta central de esta investigación es si estas empresas cuentan con un sistema de costos que sea el mismo (uniforme en el tiempo) para la mayoría o todos los productos, tenga criterios iguales y esté documentado en la estructura organizativa de la empresa. Esto implica contar con manuales de procedimientos, elaboración de reportes y procedimientos uniformes que se apliquen de igual forma independientemente del producto y del momento en que se vaya a ejecutar la actividad. Solo de esta manera se pueden obtener estimaciones de costos uniformes, confiables y representativos de la labor y los insumos que cada uno de los productos requiere durante el proceso de transformación.

¿Cuál de los dos procedimientos se aplica de forma más frecuente entre las empresas encuestadas de la muestra? En términos cuantitativos, ¿̇cuál es la probabilidad de que, si se obtiene una empresa de la muestra, esta aplique uno u otro procedimiento? Si hay alguna preferencia o tendencia por alguno de los dos métodos, la probabilidad se acercará a 0 o 1, que son los dos extremos de la escala.

\section{Posibles resultados}

- Todas las empresas (42) cuentan con un procedimiento documentado, sistematizado y de acuerdo con los cánones de la teoría de costos. De acuerdo con la definición de probabilidad como frecuencia relativa:

$\mathrm{P}$ (aplican un procedimiento sistematizado de costos) $=$ $\mathrm{n}_{1} / \mathrm{n}=42 / 42=1$ 
- $\quad$ Ninguna $\left(\mathrm{n}_{2}=0\right)$ de las empresas cuenta con un procedimiento documentado y sistematizado de acuerdo a los cánones de la teoría de costos:

$$
\begin{aligned}
& \mathrm{P}(\text { aplican un procedimiento sistematizado de costos })= \\
& \mathrm{n}_{2} / \mathrm{n}=\mathrm{O} / 4 \mathrm{O}=\mathrm{O}
\end{aligned}
$$

- $\quad$ Algunas empresas aplican un procedimiento sistematizado y otras no. Entonces:

$$
\mathrm{O} \leq \mathrm{P} \text { (aplican un procedimiento sistematizado) } \leq 1
$$

Además se cumple que: $\mathrm{n}_{1}+\mathrm{n}_{2}=\mathrm{n}$ y $\mathrm{n}_{1}, \mathrm{n}_{2} \geq 0$

Puesto que las 42 empresas se determinaron a partir de un muestreo aleatorio simple y la probabilidad de extracción de una encuesta de la muestra puede ser modelado mediante una distribución binomial, se definen las siguientes consideraciones:

- $\quad$ El experimento consiste en una sucesión de $n$ intentos o ensayos idénticos.

- En cada intento o ensayo solo son posibles dos resultados.

- $\quad$ La probabilidad de éxito $(p)$ y la probabilidad de fracaso (1-p) no cambian en el tiempo.

- Los intentos o ensayos son independientes uno del otro. El experimento se realiza usando muestreo con reemplazo.

La tabla 2 expone la definición conceptual y operacional de las variables que fueron medidas para fines de la investigación.

Tabla 2. Definiciones conceptuales y operacionales de las variables

\begin{tabular}{|l|l|l|}
\hline Variable independiente & \multicolumn{1}{|c|}{ Definición conceptual } & Definición operacional \\
\hline Materiales & $\begin{array}{l}\text { Se refieren a los costos de compra de } \\
\text { la materia prima o los materiales } \\
\text { más todos los gastos adicionales en } \\
\text { que se incurre al colocarlos en el sitio }\end{array}$ & $\begin{array}{l}\text { Es la suma de los } \\
\text { materiales utilizados en la } \\
\text { producción. }\end{array}$ \\
\hline
\end{tabular}




\begin{tabular}{|l|l|l|}
\hline Variable independiente & \multicolumn{1}{|c}{ Definición conceptual } & Definición operacional \\
\hline $\begin{array}{l}\text { para ser usados en el proceso de } \\
\text { fabricación. }\end{array}$ & \\
directos & $\begin{array}{l}\text { Están constituidos por los pagos que } \\
\text { se realizan para transformar el } \\
\text { material en el producto terminado. }\end{array}$ & $\begin{array}{l}\text { Es la suma de los salarios } \\
\text { que se pagan a los } \\
\text { operarios y obreros. }\end{array}$ \\
\hline $\begin{array}{l}\text { Gastos indirectos de } \\
\text { fabricación } \\
\text { identificados con } \\
\text { determinado producto }\end{array}$ & $\begin{array}{l}\text { Son todos los costos y gastos en que } \\
\text { se incurre en la producción pero que, } \\
\text { por su naturaleza, no son aplicables } \\
\text { directamente a esta y pueden } \\
\text { identificarse con el producto } \\
\text { terminado. }\end{array}$ & $\begin{array}{l}\text { Son los gastos que se } \\
\text { necesitan para producir, } \\
\text { pero de una manera } \\
\text { accesoria o indirecta. }\end{array}$ \\
\hline $\begin{array}{l}\text { Gastos indirectos de } \\
\text { fabricación no } \\
\text { identificados con } \\
\text { determinado producto } \\
\text { y asignados mediante } \\
\text { prorrateos }\end{array}$ & $\begin{array}{l}\text { Son todos los costos y gastos en que } \\
\text { se incurre en la producción pero que } \\
\text { por su naturaleza no son aplicables } \\
\text { directamente a esta y no pueden } \\
\text { identificarse con el producto } \\
\text { terminado. }\end{array}$ & $\begin{array}{l}\text { Son los gastos que se } \\
\text { necesitan para producir, } \\
\text { pero de una manera } \\
\text { accesoria o indirecta }\end{array}$ \\
\hline $\begin{array}{l}\text { Costos de calidad } \\
\text { Son aquellos que se realizan para que } \\
\text { el producto sea elaborado siempre } \\
\text { con la misma calidad. }\end{array}$ & $\begin{array}{l}\text { Incluyen los costos por } \\
\text { fallas internas, fallas } \\
\text { externas, reclamos, } \\
\text { garantías, etc. }\end{array}$ \\
\hline
\end{tabular}

Fuente: elaboración con base en Horngren et ál. (2014).

\section{Instrumento de medición}

Se realizó una revisión exhaustiva de los aspectos contables y financieros que deben considerar las pymes analizadas (validez de contenido). Para cada aspecto se generaron varios ítems sobre administración de costos, planeación estratégica y atribuciones económicas de las pymes en México. Esta primera versión se aplicó a una muestra piloto y se sometió a análisis de confiabilidad y de discriminación de escalas. Los ítems no correlacionados fueron eliminados.

La versión final con escala de Likert -nunca (1), casi nunca (2), en ocasiones (3), casi siempre (4), siempre (5) y o para las preguntas sin contestar- se aplicó de forma personal a 42 empresas del sector industrial solamente para resolver dudas que se tuvieran en el momento. Al recolectar los datos se numeraron en orden consecutivo los ítems de la encuesta, lo que arrojó un total de 80 constructos clasificados en tres 
secciones: identificación de los entrevistados, datos relacionados con la empresa y elementos del costo de producción.

\section{Resultados y discusión}

Tras analizar los datos, se corroboró que las pymes de transformación en Celaya y Laja Bajío no cuentan con un procedimiento sistemático de administración de costos. El 64 \% de los encuestados aseguró que la empresa tiene un sistema de costos y que este ha sido desarrollado más por costumbre y empirismo que mediante un procedimiento sistematizado. El 61 \% de los participantes refirió que la empresa no cuenta con procedimientos estructurados que incluyan manuales con métodos, procedimientos, instrucciones de trabajo y criterios mínimos de uniformidad. Afirmaron que todo es producto de la experiencia y el empirismo. Así, el 61 \% del 64 \% dice que su empresa cuenta con un sistema de costos, pero es básicamente un procedimiento no sistematizado $\left(0.61^{*} 0.64=0.39\right)$. Por tanto, se estima que solo el $39 \%$ de las empresas cuentan con un procedimiento documentado y sistematizado.

Dado que $\mathrm{n}=40, \mathrm{p}=0.39 \mathrm{y} \mathrm{n}^{*} \mathrm{p}=15.6>5$; de manera similar $\mathrm{n}=40$, (1$\mathrm{p})=0.61 \mathrm{y} \mathrm{n}^{*}(1-\mathrm{p})=24.4>5$, entonces, se usa una distribución normal para llevar a cabo la prueba de hipótesis. Considerando que la binomial se aproxima a una normal, el planteamiento matemático de las hipótesis nula y alternativa es:

Ho: $\mathrm{P}$ (no cuenta con un procedimiento sistematizado de costos) $>$ 13.80

Ha: P (puede contar con un procedimiento sistematizado de costos) $\leq 13.80$

La media de la distribución es $\mu=20$ con una varianza:

$$
\sigma^{2}=\left[n^{*} p^{*}(1-p)\right]=40^{*}\left[0.5^{*} \cdot 5\right]=10(\sigma=3.162)
$$


Se realizó una prueba de una cola con un nivel de significación de $\alpha=0.025$ y, bajo estas condiciones, el valor de "tablas" de la distribución normal es 13.80 y el valor $15.6>13.8$, por lo que se acepta Ho. No existe suficiente evidencia estadística para afirmar que la mayoría de las empresas encuestadas cuentan con un procedimiento sistemático de costos.

Es muy importante para las empresas de transformación contar con un sistema de costos que les permita determinar de una manera confiable su costo de producción, más aún en la época actual. Los ítems de la segunda parte del instrumento confirmaron de forma directa (o indirecta) la conclusión obtenida en este apartado.

Dentro de los métodos sistematizados de costos, el de su preferencia es el de costos históricos, con un 47.2 a favor. Esto se puede inferir del $61 \%$ de los que dicen contar con un método sistematizado $\left(0.472^{*} 0.61=0.287\right)$, que representa entre 11 y 12 (11.48) de las empresas de la muestra (figura 1).

Figura 1. Sistema de costos empleados

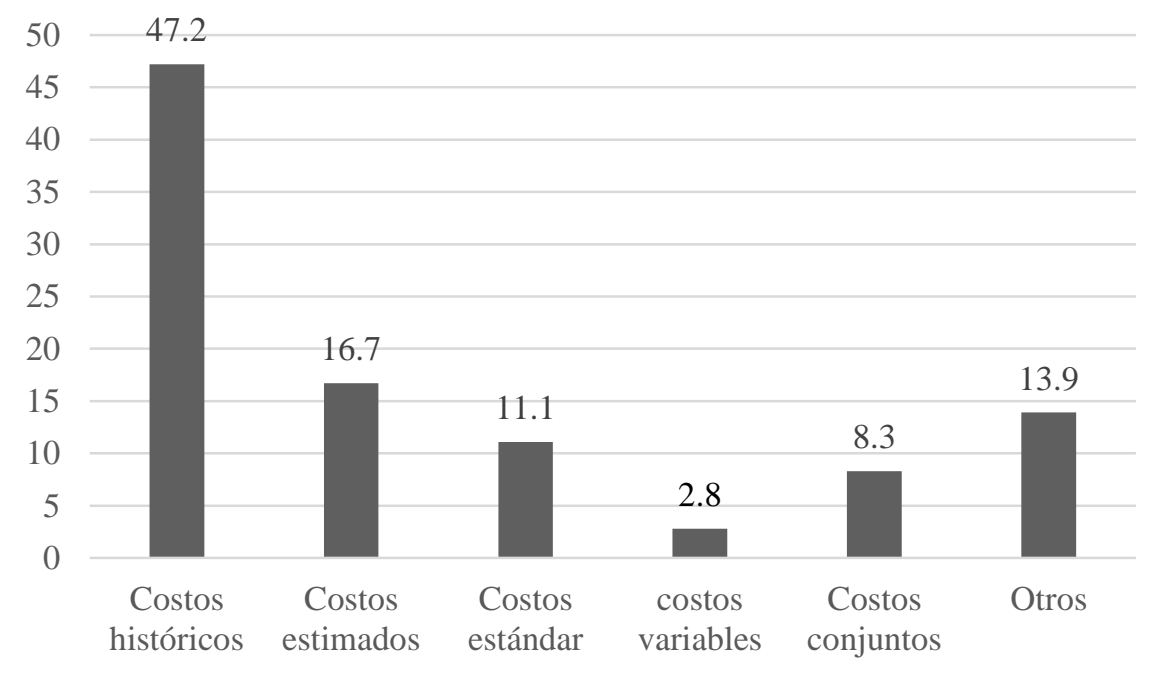

Fuente: elaboración propia. 
Este resultado es perfectamente congruente con el valor crítico de 13.8, es decir, entre 13 y 14 empresas de la muestra cuentan con un procedimiento sistemático de costos.

El 50 \% de los encuestados declaró tener poca variedad en su producción (menos de 20 productos distintos). No obstante, una buena parte de las empresas tienen un número reducido de productos y no tienen procedimientos estandarizados en las actividades críticas de los diferentes procesos. El atributo en el que se fijan las personas en cuanto a la implantación de un sistema de costos es que este sea veraz. En la tabla 3 se muestran las respuestas tabuladas de los 42 encuestados, el valor promedio y la varianza obtenidos por ítem. 


\section{Tabla 3. Resultados obtenidos de las 42 empresas encuestadas}

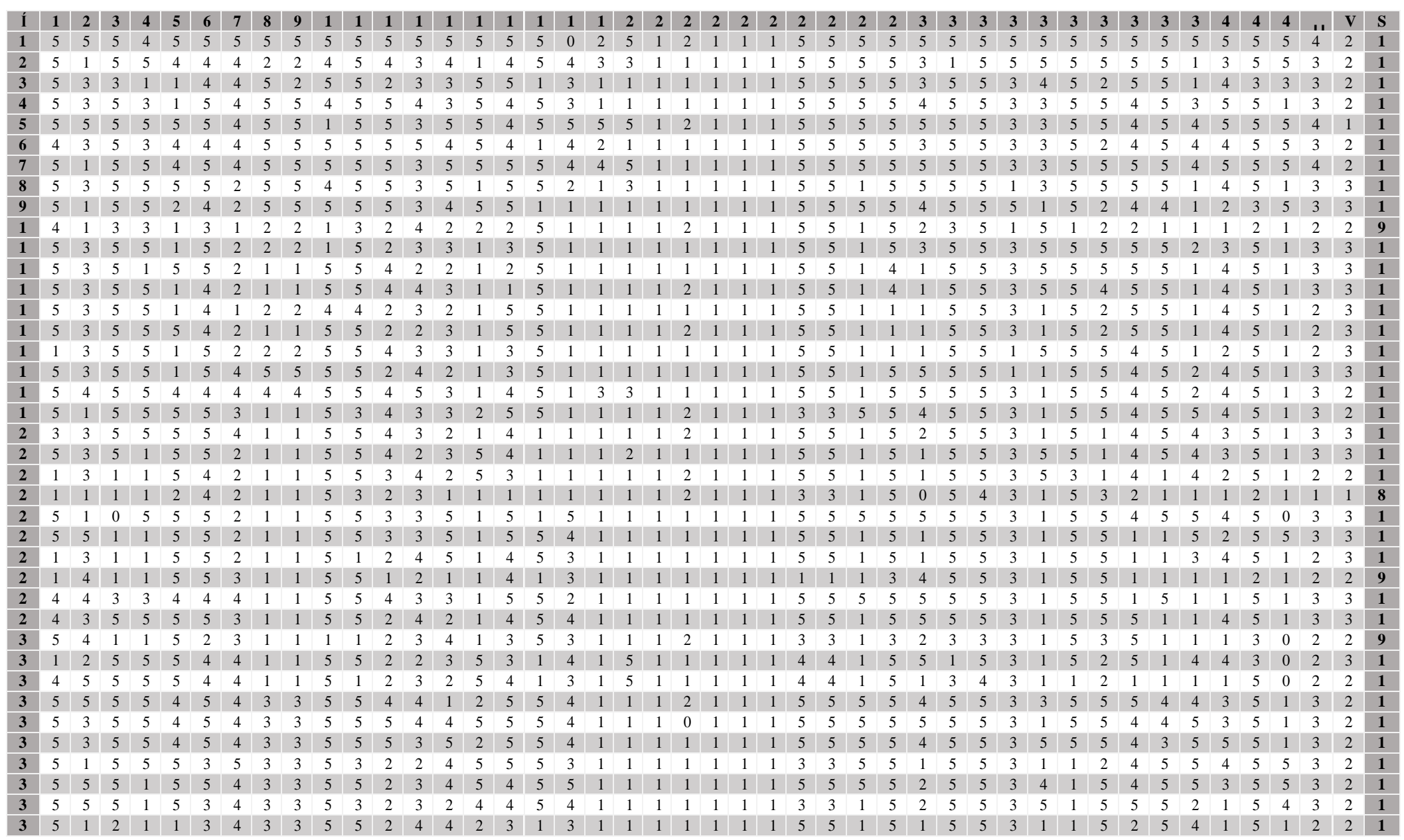

SIGNOS, Investigación en Sistemas de Gestión

ISSN: 2145-1389 | e-ISSN: 2463-1140 | DOI: https://doi.org/10.15332/24631140

Vol. 13 N.o 1 | enero-junio de 2021 


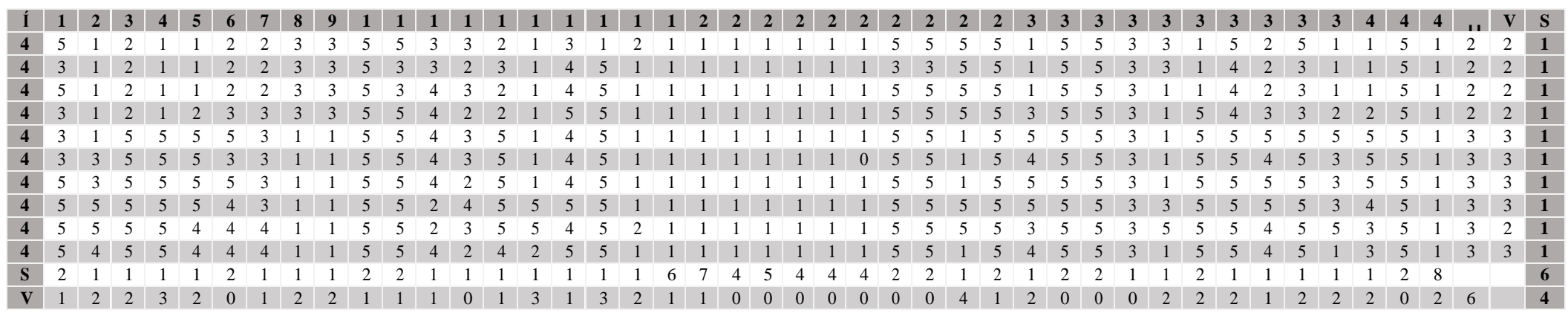

Fuente: elaboración propia.

SIGNOS, Investigación en Sistemas de Gestión

ISSN: 2145-1389 | e-ISSN: 2463-1140 | DOI: https://doi.org/10.15332/24631140

Vol. 13 N.o 1 | enero-junio de 2021 
La figura 2 muestra las calificaciones promedio obtenidas por cada ítem. La línea horizontal indica la media de las calificaciones promedio (3.158), que corresponde a "en ocasiones". Las únicas respuestas categóricas que superan la calificación 4 (casi siempre) son la 1 (considera importante que haya controles para los materiales), 5 (cuando se reciben en la empresa se revisan en cantidad) y 7 (hay en la empresa personas encargadas de recibir materiales).

Figura 2. Calificaciones obtenidas en cada ítem

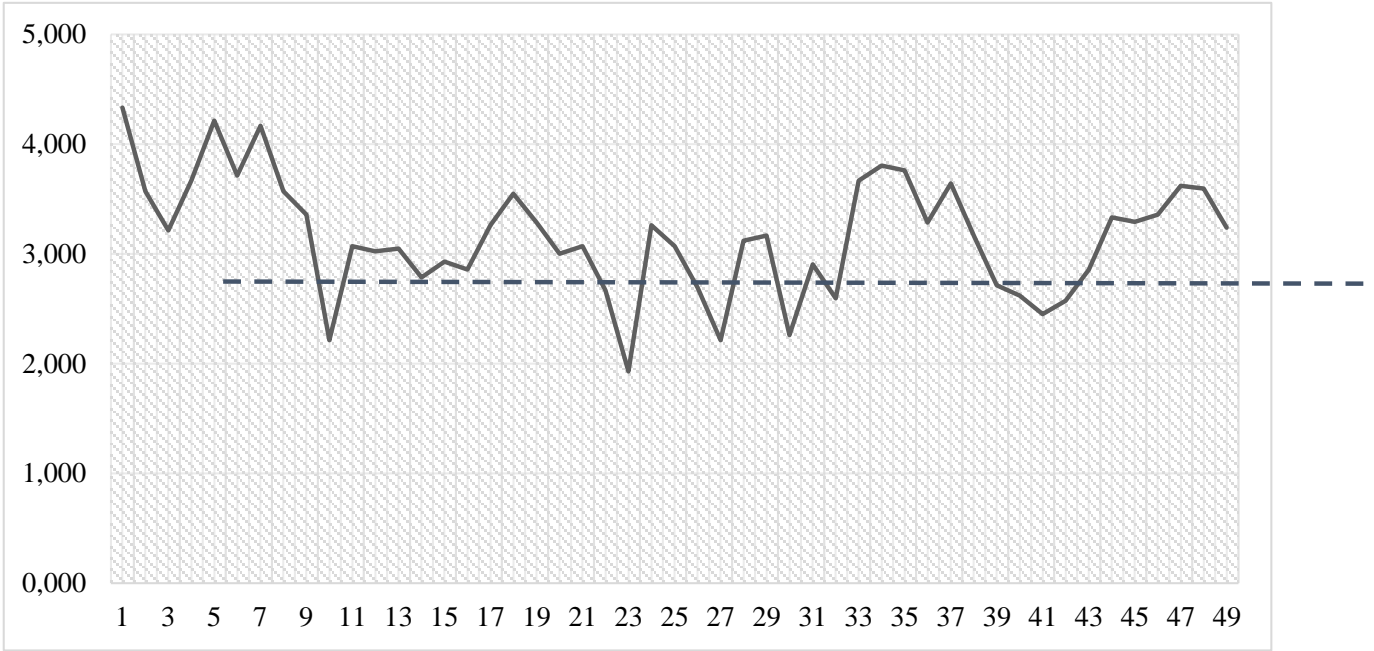

Fuente: elaboración propia.

En contraste, las preguntas que tienen asociadas la calificación cercana a 2 (casi nunca) son la 10 (la base con que se aplican a los productos los gastos indirectos cambia constantemente), la 27 (se elaboran presupuestos de los mismos) y la 37 (si el proveedor no entrega los materiales, hay un castigo para ellos). El alfa de Cronbach es de 0.87, lo cual indica que la fiabilidad del instrumento es prácticamente excelente. Los elementos diseñados para evaluar el mismo constructo generan puntuaciones similares.

De acuerdo con la opinión de Peña et ál. (2017) y en paralelo con los resultados de este estudio, se puede afirmar que en México, del $100 \%$ de 
empresas de nueva creación, sobreviven solo la mitad, debido a que al constituirse no poseen los conocimientos mínimos de administración, organización y finanzas. Uno de los problemas centrales es que ni siquiera cuentan con un sistema de costos que coadyuve a la maximización de utilidades, como se aprecia en los resultados. Cabe destacar que la Unidad de Desarrollo Productivo (Secretaría de Economía, 2020) ha promovido la reactivación de la economía de estas empresas a través de propuestas como el Fondo Nacional Emprendedor y el Programa Nacional para el Financiamiento al Microempresario.

La administración de costos es una herramienta muy valiosa que los empresarios deben usar, sobre todo al momento de iniciar una empresa o cuando se busca su crecimiento. En épocas de crisis, se vuelve mucho más importante contar con instrumentos que permitan al empresario no solo mantenerse en el mercado, sino también lograr un crecimiento sostenido. Una adecuada administración de costos no será posible si los empresarios de pymes no se actualizan en equipos, no modernizan sus procesos productivos, no planean a futuro y tampoco se sienten interesados en capacitarse o contratar los servicios de un profesional. La globalización ha abierto las puertas del país a productores extranjeros y es muy importante que, para poder competir en igualdad de condiciones, se tomen medidas tendientes a lograr lo anterior.

Arredondo (2015) menciona que una "compañía exitosa es la que sabe adónde va y tiene gente colaborando, que sabe claramente lo que se espera de ella. Adicionalmente, una compañía exitosa es la que administra sus activos y traza sus planes anticipadamente, de manera que sean previstos por el líder y este acompaña a la empresa, sin perder el rumbo bajo el cual fue creada" (p. 191). Es entonces deseable que los empresarios de pymes tengan bien determinados sus objetivos, identifiquen claramente qué 
quieren lograr, conozcan su misión y su visión, enfoquen sus esfuerzos a conseguirlas y reúnan las personas que les ayuden a esto.

La forma de clasificar las empresas varía en cada país, por lo que se recomienda homologar su método de clasificación para comparararlas de una manera más idónea. Se deben establecer criterios internacionales de categorización con base solo en el número de empleados y sin considerar el importe de las ventas. Se recomienda, también, como primer paso para el establecimiento de un plan de negocios, que las pymes definan su misión, su visión y sus objetivos, con el fin de que conozcan hacia dónde dirigir sus esfuerzos y puedan continuar con el establecimiento de su plan de negocios.

En sintonía con el modelo de gestión de costos de Nima (2018), se exhorta a los empresarios de pymes a que realicen diagramas e identifiquen los factores que pueden incrementar sus utilidades y reducir sus costos. De acuerdo con el diagrama 80-20 de Pareto, también se recomienda que identifiquen los "pocos vitales" para el crecimiento armonioso de su estructura organizacional (Dini y Stumpo, 2018).

\section{Conclusiones}

Muchas empresas no ejercen un adecuado control de sus costos de producción y esta es una de las principales causas de su cierre. Se observa que las pymes se rigen fundamentalmente por disposiciones fiscales y no, como debiera ser, por su situación financiera y una adecuada planeación de negocios.

No debiera ser por obligación fiscal que los empresarios controlen sus costos y sus inventarios. Los controles financieros internos proporcionan herramientas para facilitar la toma de decisiones y competir con mayor eficacia en los mercados nacionales e internacionales. La reforma fiscal 
mencionada es aplicable solo a personas morales y, por lo tanto, las personas físicas siguen sin tener esta obligación.

En México, las leyes fiscales imponen demasiadas obligaciones a los empresarios, por lo cual en muchas ocasiones no les queda tiempo para analizar los resultados obtenidos, ya que solo están pensando en cómo van a conseguir el dinero para el pago de sus impuestos. Se recomienda que el Estado facilite el cumplimiento de las obligaciones fiscales y dé más tiempo a los empresarios para analizar los resultados obtenidos en sus empresas.

También se considera necesario que los contadores tomen más conciencia de la importancia de la administración de los costos y apoyen más a los empresarios en esta tarea, ya que actualmente se dedican principalmente al cálculo de impuestos y dejan al empresario solo en la administración de sus negocios. Mediante una adecuada administración de los costos es posible lograr ventajas competitivas en las pequeñas y medianas empresas de México.

Entre las limitantes del proyecto se señala la falta de confianza de algunos empresarios, pues consideraron la información solicitada de carácter confidencial y no visualizaron los beneficios que se les expusieron al comenzar el trabajo.

\section{Referencias}

Arredondo, M. G. (2015). Análisis de las mejores prácticas empresariales en las áreas de administración, mercadotecnia, manufactura y desarrollo organizacional. Un estudio de caso [Tesis doctoral no publicada]. Universidad de Celaya.

Banco Mundial. (2016, 20 de junio). Los emprendedores y las pequeñas empresas impulsan al crecimiento económico y crean empleos. https://www.bancomundial.org/es/news/feature/2016/06/20/entrepreneursand-small-businesses-spur-economic-growth-and-create-jobs 
Calleja, F. (2018). Costos (2. ${ }^{\mathrm{a}}$ ed.). Pearson Educación; Universidad de Sonora.

Dini, M. y Stumpo, G. (Coords.). (2018). Mipymes en América Latina: un frágil desempeño y nuevos desafíos para las políticas de fomento. Documentos de proyectos (LC/TS.2018/75). Comisión Económica para América Latina y el Caribe. https://repositorio.cepal.org/bitstream/handle/11362/44148/1/S1800707_es.pdf

Guizar, R. (2007). Desarrollo organizacional (2. ${ }^{\mathrm{a}}$ ed.). McGraw Hill Interamericana.

Hodgson, P. y White, R. (2002). Incertidumbre empresarial. Cómo fijar el rumbo cuando el rumbo cambia. Prentice Hall.

Horngren, Ch., Datar, S. y Rajan, M. (2016). Contabilidad de costos. Un enfoque gerencial. (14. ${ }^{\mathrm{a}}$ ed.). Prentice Hall Hispanoamericana.

Instituto Nacional de Estadística y Geografía. (2019, 2 de septiembre). Inegi presenta resultados de la Encuesta Nacional sobre Productividad y Competitividad de las Micro, Pequeñas y Medianas Empresas (Enaproce) 2018 [comunicado de prensa n. $\left.{ }^{\circ} 448 / 19\right]$.

https://www.inegi.org.mx/contenidos/saladeprensa/boletines/2019/especiales/E NAPROCE2018.pdf

Nima, J. (2018). Propuesta de un proceso de costos dentro de un modelo de gestión para Mype del subsector restaurantes en Lima Metropolitana [Tesis de pregrado, Universidad Peruana de Ciencias Aplicadas]. Repositorio Académico UPC. http://hdl.handle.net/10757/624476

Ojeda, J. F. (2013). Análisis del clima psicológico y su relación con los estilos de pensamiento bajo la perspectiva de la teoría HBDI [Tesis doctoral no publicada]. Universidad de Celaya.

Peña, N., Aguilar, O. y Posada, R. (2017). Factores que determinan el cierre de la micro y pequeña empresa. Comparativo entre empresas activas e inactivas en México y Colombia. Pearson Educación.

Ramírez, D. (2018). Contabilidad administrativa (9. ${ }^{\mathrm{a}}$ ed.). Mc-Graw-Hill.

Secretaría de Economía. (2020, 31 de marzo). Unidad de Desarrollo Productivo (UDP). Gobierno de México. https://www.gob.mx/se/acciones-y-programas/unidad-dedesarrollo-productivo

SIGNOS, Investigación en Sistemas de Gestión

ISSN: 2145-1389 | e-ISSN: 2463-1140 | DOI: https://doi.org/10.15332/24631140

Vol. 13 N. ${ }^{\circ} 1$ | enero-junio de 2021 